\title{
Granulocyte Differentiation
}

National Cancer Institute

\section{Source}

National Cancer Institute. Granulocyte Differentiation. NCI Thesaurus. Code C96831.

The biological process that refers to the maturation of granulocytes. 